\title{
DOMAINS WHICH ARE LOCALLY UNIFORMLY LINEARLY CONVEX IN THE KOBAYASHI DISTANCE
}

\author{
MONIKA BUDZYŃSKA
}

Received 2 October 2001

We show a construction of domains in complex reflexive Banach spaces which are locally uniformly convex in linear sense in their Kobayashi distance. We also show connections between norm and Kobayashi distance properties.

\section{Introduction}

Recently, in [1], it has been proved that if $B$ is an open unit ball in a Cartesian product $l^{2} \times l^{2}$ furnished with the $l^{p}$-norm $\|\cdot\|$ and $k_{B}$ is the Kobayashi distance on $B$, then the metric space $\left(B, k_{B}\right)$ is locally uniformly convex in linear sense. Our construction of domains, which are locally uniformly convex in their Kobayashi distances, is based on the ideas from [1]. Such domains play an important role in the fixed-point theory of holomorphic mappings (see $[1,2,4,13$, 14]).

In Section 4, we show connections between norm and Kobayashi distance properties.

\section{Preliminaries}

Throughout this paper, all Banach spaces $X$ will be complex and reflexive, all domains $D \subset X$ bounded and convex, and $k_{D}$ will denote the Kobayashi distance on $D[6,7,9,10,11,12]$.

We will use the notions and notations from [2]. Here, we recall a few facts only.

The Kobayashi distance $k_{D}$ is locally equivalent to the norm $\|\cdot\|$ [9]. Indeed, if $\operatorname{dist}_{\|\cdot\|}(x, \partial D)$ denotes the distance in $(X,\|\cdot\|)$ between the point $x$ and the boundary $\partial D$ of the domain $D$, and $\operatorname{diam}_{\|\cdot\|} D$ is the diameter of $D$ in $(X,\|\cdot\|)$, 
514 Locally uniformly linearly convex domains

then

$$
\operatorname{argtanh}\left(\frac{\|x-y\|}{\operatorname{diam}_{\|\cdot\|} D}\right) \leq k_{D}(x, y)
$$

for all $x, y \in D$ and

$$
k_{D}(x, y) \leq \arg \tanh \left(\frac{\|x-y\|}{\operatorname{dist}_{\|\cdot\|}(x, \partial D)}\right)
$$

whenever $\|x-y\|<\operatorname{dist}_{\|\cdot\|}(x, \partial D)$.

A subset $C$ of $D$ is said to lie strictly inside $D$ if $\operatorname{dist}_{\|\cdot\|}(C, \partial D)>0$. We can observe that a subset $C$ of $D$ is $k_{D}$-bounded if and only if $C$ lies strictly inside $D$ [9, Proposition 23].

Each open (closed) $k_{D}$-ball in the metric space $\left(D, k_{D}\right)$ is convex [15] and if $D$ is strictly convex, then every $k_{D}$-ball is also strictly convex in a linear sense $[3,18]$ ( see also [17]).

The metric space $\left(D, k_{D}\right)$ is called a locally uniformly linearly convex space [2] if there exist $w \in D$ and the function

$$
\delta(w, \cdot, \cdot, \cdot, \cdot, \cdot)
$$

such that for all $0<R_{1}, k_{D}(w, z) \leq R_{1}, 0<R_{2} \leq R \leq R_{3}$, and $0<\epsilon_{1} \leq \epsilon \leq \epsilon_{2}<2$, we have

$$
\left.\begin{array}{l}
\quad \delta\left(w, R_{1}, R_{2}, R_{3}, \epsilon_{1}, \epsilon_{2}\right)>0 \\
k_{D}(z, x) \leq R \\
k_{D}(z, y) \leq R \\
k_{D}(x, y) \geq \epsilon R
\end{array}\right\} \Longrightarrow k_{D}\left(z, \frac{1}{2} x+\frac{1}{2} y\right) \leq\left(1-\delta\left(w, R_{1}, R_{2}, R_{3}, \epsilon_{1}, \epsilon_{2}\right)\right) R
$$

The function $\delta(w, \cdot, \cdot, \cdot, \cdot, \cdot)$ is called a modulus of linear convexity for the Kobayashi distance $k_{D}$.

The open unit ball $B_{H}$ in a Hilbert space is called the Hilbert ball $[5,7,8,14$, 16].

For more useful properties of the Kobayashi distance, see [14].

\section{Examples of locally uniformly linearly convex domains}

The first known domain is the Hilbert ball $[13,14]$. Other examples are given in [1]. Namely, if $B$ is the open unit ball in a Cartesian product $l^{2} \times l^{2}$ furnished with the $l^{p}$-norm, where $1<p<\infty$ and $p \neq 2$, then the metric space $\left(B, k_{B}\right)$ is also locally uniformly linearly convex.

Before stating our main result, we prove the following auxiliary lemma.

Lemma 3.1. Let $X$ be a finite-dimensional Banach space and $D$ a bounded, closed, and strictly convex domain in $X$. Then, the metric space $\left(D, k_{D}\right)$ is locally uniformly linearly convex. 
Proof. Since $D$ is a bounded and strictly convex domain in $X$, each $k_{D}$-ball is strictly convex in a linear sense. Therefore, using the equivalent definition of the $k_{D}$-boundedness and the compactness argument, we see that the metric space $\left(D, k_{D}\right)$ is locally uniformly linearly convex.

Now, we state the main result of this paper.

Theorem 3.2. Let $Y$ be a finite-dimensional subspace of a complex reflexive $B a$ nach space $X$ and $D$ a bounded strictly convex domain in $X$. Suppose that

(i) there exists a point $x_{0} \in D_{0}=D \cap Y$,

(ii) there exists a holomorphic retraction $r: D \rightarrow D_{0}$,

(iii) for every $R>0$ and for any three points $x, y$, and $z$ in the closed $k_{D}$-ball $\bar{B}\left(x_{0}, R\right)$, there exists a biholomorphic affine mapping $T: D \rightarrow D$ such that $T\left(x_{0}\right)=x_{0}$ and $T(x), T(y), T(z) \in Y \cap D_{0}$.

Then, the metric space $\left(D, k_{D}\right)$ is locally uniformly linearly convex.

Proof. First, observe that $D_{0}$ is a strictly convex domain in $Y$ and by (ii),

$$
k_{D_{0}}(u, w)=k_{D}(u, w)
$$

for all $u, w \in D_{0}$. This (combined with assumption (i)) implies that the closed $k_{D_{0}}$-ball $\overline{B_{0}}\left(x_{0}, R\right)$ is equal to $\bar{B}\left(x_{0}, R\right) \cap D_{0}$.

Let $x, y$, and $z$ be three arbitrarily chosen points in the closed $k_{D}$-ball $\bar{B}\left(x_{0}, R\right)$. By assumption (iii), there exists a biholomorphic affine mapping $T: D \rightarrow D$ such that $T x, T y, T z \in Y \cap D_{0}$ and $T x_{0}=x_{0}$. Since this biholomorphic mapping is always a $k_{D}$-isometry $[6,7,9,10,14]$, we get

$$
\begin{aligned}
T x, T y, T z & \in \bar{B}\left(x_{0}, R\right) \cap D_{0}, \\
k_{D}(x, y) & =k_{D_{0}}(T x, T y), \\
k_{D}(x, z) & =k_{D_{0}}(T x, T z), \\
k_{D}(y, z) & =k_{D_{0}}(T y, T z) .
\end{aligned}
$$

Therefore, we may restrict our further considerations to the finite-dimensional Banach space $Y$. By Lemma 3.1, the metric space $\left(D_{0}, k_{D_{0}}\right)$ is locally uniformly linearly convex and this implies the same property of $\left(D, k_{D}\right)$.

Example 3.3. If $B$ is the open unit ball in a Cartesian product $X=\mathbb{C}^{n} \times l^{2}$, furnished with the $l^{p}$-norm, where $1<p<\infty$, and in $\mathbb{C}^{n}$ we have a strictly convex norm (i.e., the open unit ball in this norm is strictly convex), then the metric space $\left(B, k_{B}\right)$ is locally uniformly linearly convex.

Indeed, let $\left\{e_{1}, e_{2}, \ldots\right\}$ be the standard basis in the Hilbert space $l^{2}$. For any three points $x=\left(x_{1}, x_{2}\right), y=\left(y_{1}, y_{2}\right)$, and $z=\left(z_{1}, z_{2}\right)$ in $B \subset \mathbb{C}^{n} \times l^{2}$, there exists a linear isometry $T_{1}: l^{2} \rightarrow l^{2}$ such that

$$
\tilde{T} x_{2}, \tilde{T} y_{2}, \tilde{T} z_{2} \in \operatorname{lin}\left\{e_{1}, e_{2}, e_{3}\right\}
$$


516 Locally uniformly linearly convex domains

Put

$$
\begin{gathered}
Y=\mathbb{C}^{n} \times \operatorname{lin}\left\{e_{1}, e_{2}, e_{3}\right\}, \\
B_{1}=Y \cap B, \\
T\left(w_{1}, w_{2}\right)=\left(w_{1}, \tilde{T} w_{2}\right)
\end{gathered}
$$

for $\left(w_{1}, w_{2}\right) \in B \subset \mathbb{C}^{n} \times l^{2}$. It is obvious that $B_{1}$ is the open unit ball in $Y$ and

$$
k_{B}(u, w)=k_{B_{1}}(u, w)
$$

for all $u, w \in B_{1}$. Therefore, we can apply Theorem 3.2.

Example 3.4. In the Cartesian product $X=l^{2} \times l^{2} \times l^{2}$, we have the following norm:

$$
\left\|\left(x_{1}, x_{2}, x_{3}\right)\right\|=\left[\left\|x_{1}\right\|^{p}+\left(\left\|x_{2}\right\|^{q}+\left\|x_{3}\right\|^{q}\right)^{p / q}\right]^{1 / p}
$$

where $1<p, q<\infty, p, q \neq 2, p \neq q$, and $\left(x_{1}, x_{2}, x_{3}\right) \in X$. Let $B$ be the open unit ball in $X$. The metric space $\left(B, k_{B}\right)$ is locally uniformly linearly convex. The proof of this fact is similar to that given in Example 3.3.

Example 3.5. Let $X$ be the Hilbert space $l^{2}$ with the standard orthonormal basis $\left\{e_{1}, e_{2}, \ldots\right\}$. Let $D_{0}$ be an arbitrary bounded strictly convex domain in $\operatorname{lin}\left\{e_{1}\right\}$. Let $\partial D_{0}$ denote the boundary of $D_{0}$ in $\operatorname{lin}\left\{e_{1}\right\}$. A strictly convex domain $D \in X$, generated by $D_{0}$, is defined as follows:

$$
D=\left\{z+w: z \in D_{0}, w \in \operatorname{lin}\left\{e_{2}, e_{3}, \ldots\right\},\|w\|<\sqrt{\operatorname{dist}\left(z, \partial D_{0}\right)}\right\}
$$

It is easy to check that we may apply Theorem 3.2, and therefore the metric space $\left(D, k_{D}\right)$ is locally uniformly linearly convex.

Remark 3.6. A construction of more complicated examples is obvious.

\section{Connections between norm and Kobayashi distance properties}

There is some connection between the local uniform convexity in linear sense of the unit ball $\left(B, k_{B}\right)$ and the uniform convexity of the whole Banach space. Namely, the following theorem is valid.

Theorem 4.1. Let $(X,\|\cdot\|)$ be a complex Banach space and $B$ the open unit ball in $(X,\|\cdot\|)$. If $\left(B, k_{B}\right)$ is locally uniformly convex in linear sense, then the Banach space $(X,\|\cdot\|)$ is uniformly convex. 
Proof. It is sufficient to show that the ball $B(0,1 / 2)$ in $(X,\|\cdot\|)$ is uniformly convex. Let

$$
\begin{gathered}
\|x\|=\|y\|=\frac{1}{2}, \\
\|x-y\| \geq \frac{1}{2} \epsilon .
\end{gathered}
$$

We know that the norm $\|\cdot\|$ and the Kobayashi distance are locally equivalent and, additionally, we have

$$
\begin{gathered}
k_{B}(0, x)=k_{B}(0, y)=\operatorname{argtanh}\left(\frac{1}{2}\right)=R, \\
k_{B}(x, y) \geq \operatorname{argtanh}\left(\frac{\|x-y\|}{2}\right) \geq \frac{\operatorname{argtanh}((1 / 4) \epsilon)}{R} R=\eta R .
\end{gathered}
$$

Hence, by the local uniform convexity in linear sense of the unit ball $\left(B, k_{B}\right)$, we get

$$
\begin{aligned}
k_{B}\left(0, \frac{1}{2} x+\frac{1}{2} y\right) & \leq(1-\delta(0, R, R, R, \eta, \eta)) R \\
& =(1-\delta(0, R, R, R, \eta, \eta)) \arg \tanh \left(\frac{1}{2}\right) \\
& =\operatorname{argtanh}\left(\left(1-\delta^{*}\right) \frac{1}{2}\right)
\end{aligned}
$$

and therefore

$$
\left\|\frac{1}{2} x+\frac{1}{2} y\right\| \leq\left(1-\delta^{*}\right) \frac{1}{2}
$$

where

$$
\delta^{*}=1-2 \tanh \left((1-\delta(0, R, R, R, \eta, \eta)) \arg \tanh \left(\frac{1}{2}\right)\right) .
$$

Remark 4.2. There is the following open problem. Does the uniform convexity of the complex Banach space $(X,\|\cdot\|)$ imply the local uniform convexity in linear sense of $\left(B, k_{B}\right)$, where $B$ is the open unit ball in $(X,\|\cdot\|)$ ?

It is worth recalling here two facts about strict convexity. As we mentioned in Section 2, the strict convexity of the domain $D$ implies that every $k_{D}$-ball is also strictly convex in a linear sense $[3,18]$ (see also [17]). It is natural to ask whether the strict convexity of $\left(D, k_{D}\right)$ implies the strict convexity of $D$. The answer is, no, as the following example shows. 
Example 4.3 (see [4]). Consider the domain

$$
D=\Delta \cap\left\{z \in \mathbb{C}: \operatorname{Re} z<\frac{1}{\sqrt{2}}\right\}
$$

in the complex plane $\mathbb{C}$. Then, every $k_{D}$-ball is strictly convex in a linear sense but $D$ is not a strictly convex set.

On the other hand, in the case of the open unit ball, we have the positive answer to the above question.

Theorem 4.4. Let $(X,\|\cdot\|)$ be a complex Banach space and $B$ the open unit ball in $(X,\|\cdot\|)$. The Banach space $(X,\|\cdot\|)$ is strictly convex if and only if $\left(B, k_{B}\right)$ is strictly convex in linear sense.

Proof. We know that the strict convexity of the ball $B$ implies that every $k_{B}$-ball is also strictly convex in a linear sense $[3,18]$ (see also [17]). Now, if each $k_{B}$-ball is strictly convex in a linear sense, then we can repeat the method of the proof of Theorem 4.1 to get the strict convexity of the Banach space $(X,\|\cdot\|)$.

\section{References}

[1] M. Budzyńska, An example in holomorphic fixed point theory, to appear in Proc. Amer. Math. Soc.

[2] Local uniform linear convexity with respect to the Kobayashi distance, to appear in Abstr. Appl. Anal.

[3] M. Budzyńska and T. Kuczumow, A strict convexity of the Kobayashi distance, Fixed Point Theory and Applications (Y. J. Cho, ed.), vol. 4, Nova Science Publishers, New York, to appear.

[4] M. Budzyńska, T. Kuczumow, and A. Stachura, Properties of the Kobayashi distance, Proceedings of the International Conference on Nonlinear Analysis and Convex Analysis (Hirosaki, 2001), to appear.

[5] S. Dineen, The Schwarz Lemma, Oxford Mathematical Monographs, Oxford University Press, New York, 1989.

[6] T. Franzoni and E. Vesentini, Holomorphic Maps and Invariant Distances, Notas de Matemática, vol. 69, North-Holland Publishing, Amsterdam, 1980.

[7] K. Goebel and S. Reich, Uniform Convexity, Hyperbolic Geometry, and Nonexpansive Mappings, Monographs and Textbooks in Pure and Applied Mathematics, vol. 83, Marcel Dekker, New York, 1984.

[8] K. Goebel, T. Sekowski, and A. Stachura, Uniform convexity of the hyperbolic metric and fixed points of holomorphic mappings in the Hilbert ball, Nonlinear Anal. 4 (1980), no. 5, 1011-1021.

[9] L. A. Harris, Schwarz-Pick systems of pseudometrics for domains in normed linear spaces, Advances in Holomorphy (Proc. Sem. Univ. Fed. Rio de Janeiro, Rio de Janeiro, 1977), North-Holland Math. Stud., vol. 34, North-Holland Publishing, Amsterdam, 1979, pp. 345-406.

[10] M. Jarnicki and P. Pflug, Invariant Distances and Metrics in Complex Analysis, de Gruyter Expositions in Mathematics, vol. 9, Walter de Gruyter, Berlin, 1993. 
[11] S. Kobayashi, Invariant distances on complex manifolds and holomorphic mappings, J. Math. Soc. Japan 19 (1967), 460-480.

[12] _ Hyperbolic Manifolds and Holomorphic Mappings, Pure and Applied Mathematics, vol. 2, Marcel Dekker, New York, 1970.

[13] T. Kuczumow, Fixed points of holomorphic mappings in the Hilbert ball, Colloq. Math. 55 (1988), no. 1, 101-107.

[14] T. Kuczumow, S. Reich, and D. Shoikhet, Fixed points of holomorphic mappings: a metric approach, Handbook of Metric Fixed Point Theory, Kluwer Academic Publishers, Dordrecht, 2001, pp. 437-515.

[15] T. Kuczumow and A. Stachura, Iterates of holomorphic and $k_{D}$-nonexpansive mappings in convex domains in $\mathbf{C}^{n}$, Adv. Math. 81 (1990), no. 1, 90-98.

[16] W. Rudin, Function Theory in the Unit Ball of $\mathbf{C}^{n}$, Grundlehren der Mathematischen Wissenschaften, vol. 241, Springer-Verlag, New York, 1980.

[17] J.-P. Vigué, La métrique infinitésimale de Kobayashi et la caractérisation des domaines convexes bornés [The Kobayashi infinitesimal metric and the characterization of bounded convex domains], J. Math. Pures Appl. (9) 78 (1999), no. 9, 867-876 (French).

[18] _ Stricte convexité des domaines bornés et unicité des géodésiques complexes [Strict convexity of bounded domains and uniqueness of complex geodesics], Bull. Sci. Math. 125 (2001), no. 4, 297-310 (French).

Monika Budzyńska: Instytut Matematyki, Uniwersytet M. Curie-Sklodowskiej (UMCS), 20-031 Lublin, Poland; Instytut Matematyki Panstwowa Wyzsza Szkola Zawodowa (PWSZ), 20-120 Chełm, Poland

E-mail address: monikab@golem.umcs.lublin.pl 


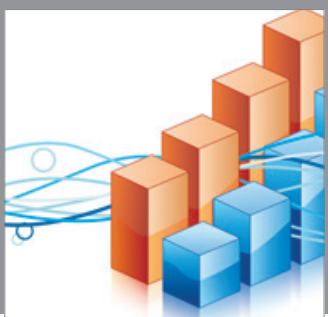

Advances in

Operations Research

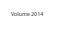

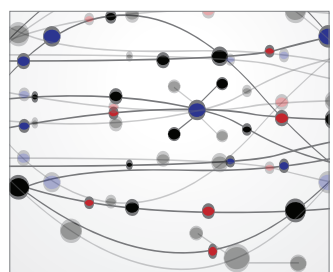

\section{The Scientific} World Journal
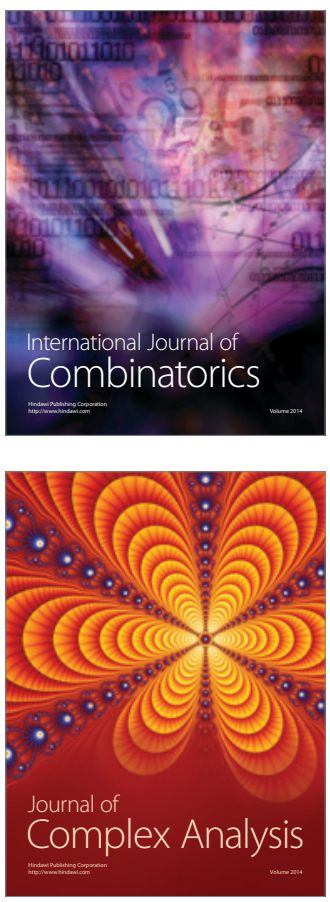

International Journal of

Mathematics and

Mathematical

Sciences
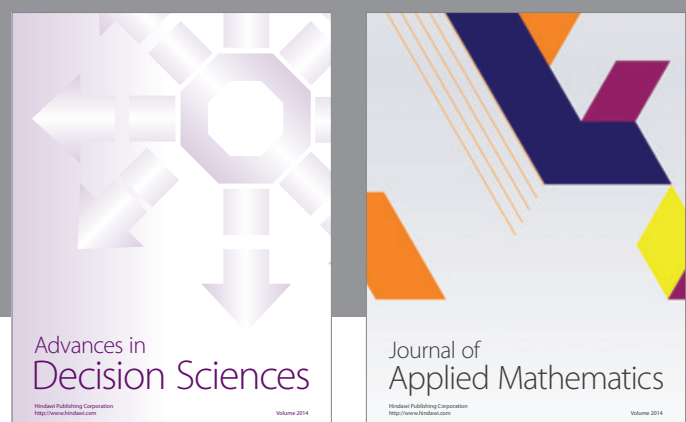

Journal of

Applied Mathematics
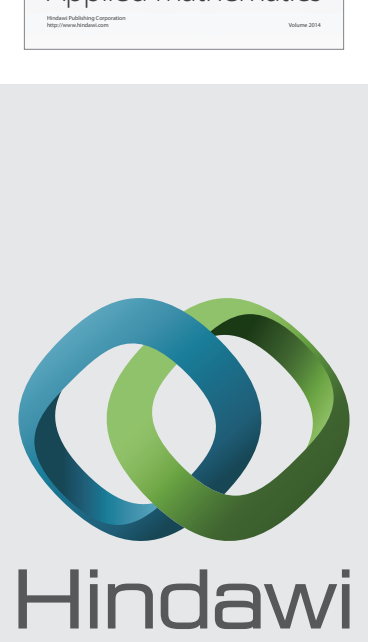

Submit your manuscripts at http://www.hindawi.com
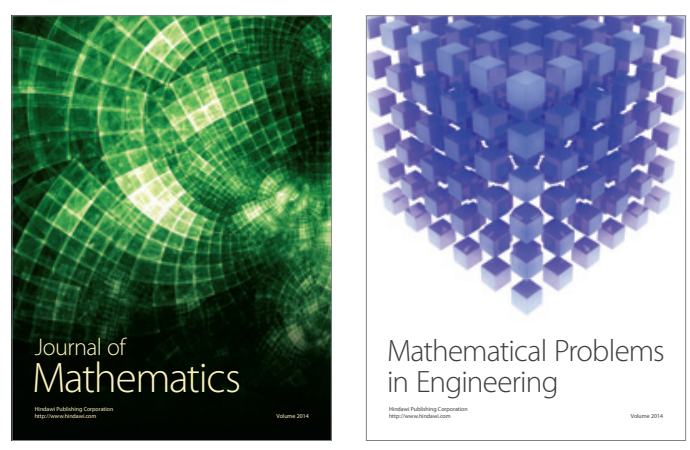

Mathematical Problems in Engineering
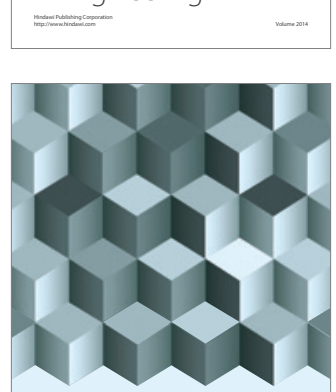

Journal of

Function Spaces
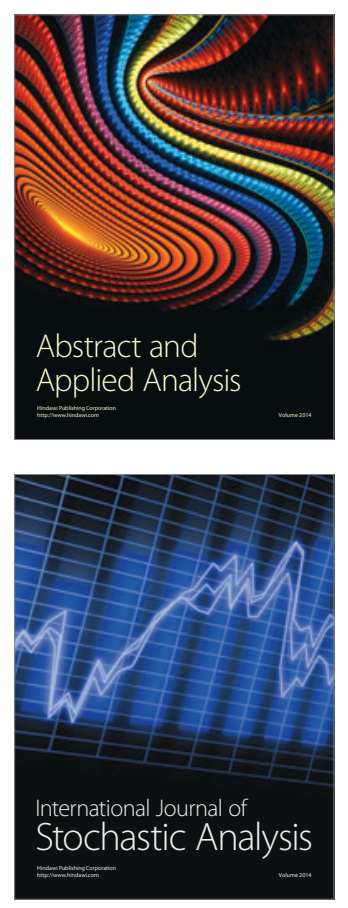

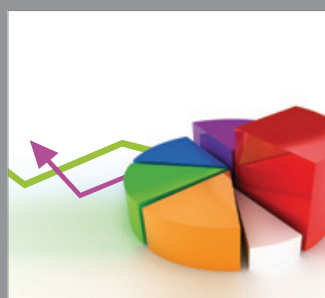

ournal of

Probability and Statistics

Promensencen
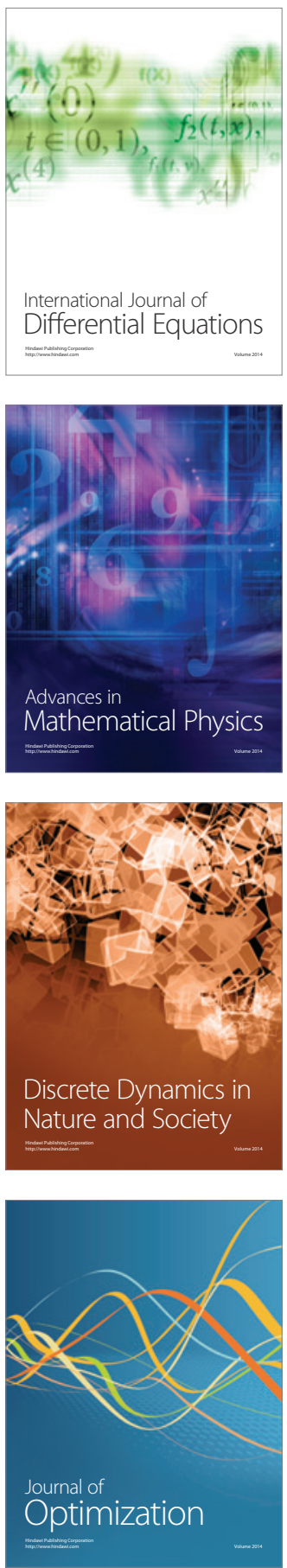